\title{
Plasma-seq: a novel strategy for metastatic prostate cancer analysis
}

\author{
Caitlin Farris and Jeffrey M Trimarchi* \\ See related research article: http://genomemedicine.com/content/5/4/30
}

\begin{abstract}
Personalized genomics will only be useful for monitoring the prognosis of patients with cancer when it becomes much more cost-effective and quicker to apply. A recent study brings this closer to reality with the development of plasma-seq, a rapid, low-cost method that sequences the circulating DNA present in the peripheral blood of patients with cancer. The power of this technique is demonstrated with the examination of tumor genomes from patients with prostate cancer.
\end{abstract}

\section{Introduction}

Prostate cancer is prevalent throughout the world. It is the most common cancer in European men and the second most common in American men [1]. In the United States, prostate cancer presents a significant health challenge; each year, 200,000 new cases are reported and 32,000 men die of the disease [2]. Historically, there has been a shift toward early stage diagnosis of prostate tumors using the prostate-specific antigen test. This test was intended to enable earlier treatment of patients and a better outcome. However, prostate cancer frequently metastasizes or recurs, even after early treatment [3]. In addition, owing to limitations associated with the prostate-specific antigen test, its use is controversial and in many instances no longer recommended [4].

The current standard therapy for patients with metastatic or recurrent prostate cancer involves the reduction of androgen levels [1]. Under this androgen-deprivation therapy, tumor progression can be stopped for several years, but eventually disease progression resumes, albeit more slowly. This castration-resistant prostate cancer (CRPC) is notoriously heterogeneous in terms of

*Correspondence: jtrimarc@iastate.edu

Department of Genetics, Development and Cell Biology, 2114 Molecular Biology, lowa State University, Ames, IA 50011, USA response to treatment and patient survival [1]. Therefore, a better understanding of the disease and its response to treatments is urgently required.

\section{Challenges in prostate tumor analysis}

Studying tumor tissue from patients with metastatic prostate cancer is challenging, as it tends to spread to the bone, making biopsies difficult. Even the primary tumors themselves are small and mixed in with stromal tissue, and so precise dissection is challenging [5]. Despite these obstacles, researchers have undertaken whole-exome and whole-genome sequencing studies of CRPC cases in an effort to reveal the genetics changes in these patients $[5,6]$. Studies such as these found no single gene mutated across all prostate cancers, but did find alterations such as the fusion (TMPRSS2-ERG) between the transmembrane protease, serine 2 gene (TMPRSS2) and the ETS related gene $(E R G)$ in about half of the tumors [6]. Additionally, low overall somatic point mutation rates were consistently observed when compared with other tumors such as glioblastomas $[5,6]$.

Because acquiring tumor tissue is problematic, other approaches are needed. One such approach, blood-based assays, offers distinct advantages. These assays are only minimally invasive, do not require intricate dissections and are, therefore, more straightforward in their standardization, allowing for repeated sampling over time. Studies using whole blood have shown that mRNA expression signatures can be used to group patients with CRPC into high- and low-risk groups [7]. Circulating tumor cells (CTCs) are rare in the peripheral blood of patients with cancer, including those with metastatic prostate cancer [8]. These cells have been isolated and used to identify molecular markers of prostate cancer, as well as its response to treatments [8]. While informative, these studies are limited by their reliance on the isolation of CTCs through the expression of epithelial cell adhesion molecule [8]. Therefore, this technique requires additional expenses involving reagents and equipment beyond just a simple blood draw [8]. In addition, any CTCs that are epithelial cell adhesion molecule-negative will be missed by this assay. 


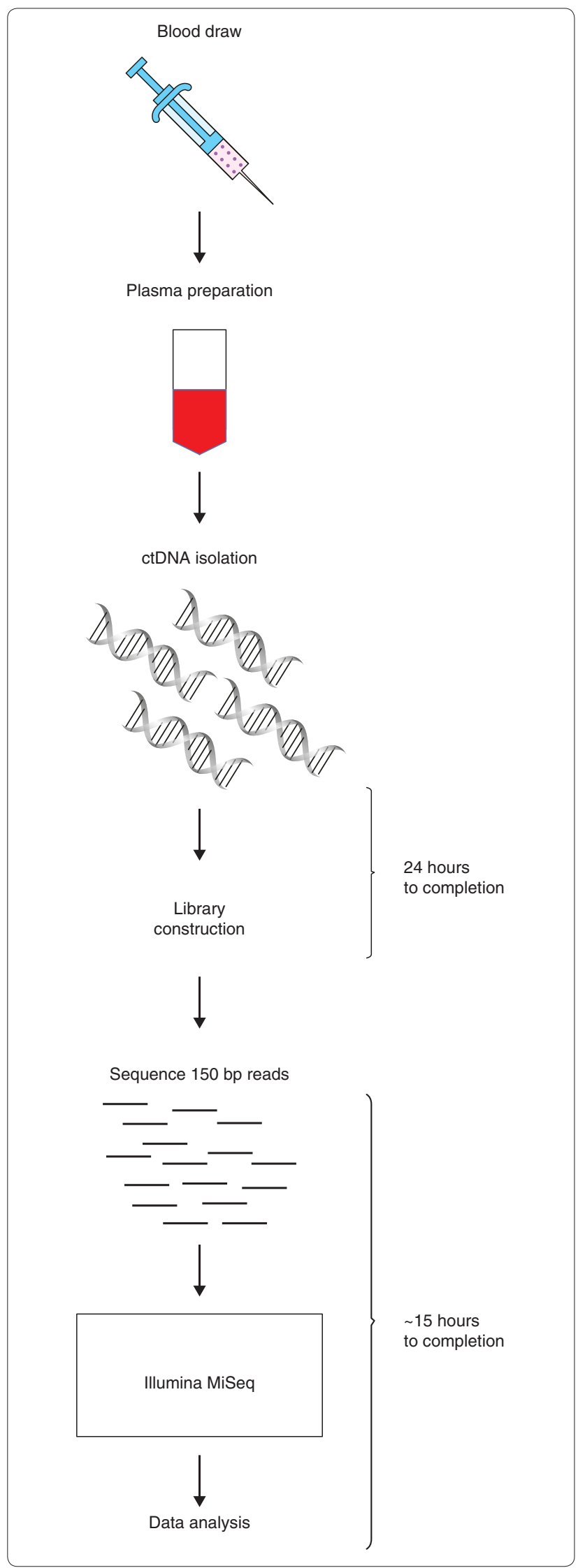

Fragments of circulating tumor DNA (ctDNA), 140 base pairs (bp) to $170 \mathrm{bp}$ long, have been found at low numbers in the blood of patients with tumors [9]. Analysis of this plasma DNA provides a new option for the analysis of tumor genomes. It can be used to identify recurring mutations in subsets of patients with cancer. Previously, ctDNA from ovarian cancer has been amplified and sequenced using a tagged amplicon method called TAm-seq [9]. This technique had excellent sensitivity as demonstrated by its ability to sequence DNA regions present in a single copy of ctDNA [9]. Altogether, this 'liquid biopsy' approach shows great potential and, with standardization and a reduction in price, could revolutionize personal genomics in cancer management.

\section{Plasma-seq}

To bring the power of whole-genome analysis to a more routine clinical benchtop setting, Heitzer et al. [10] have now developed an improved technique that profiles cellfree plasma DNA from patients (Figure 1). This new method, which the authors name plasma-seq, uses shallow sequencing (depth of about $0.1 \times$ ) with a benchtop highthroughput instrument (Illumina MiSeq; Illumina, Inc., San Diego, CA, USA) to examine the tumor genomes of patients with metastasized prostate cancer. Before implementing plasma-seq on samples from these patients, Heitzer et al. thoroughly tested their approach on different samples, including fragmented DNA from a colon cancer cell line and samples from pregnancies with trisomies of distinct chromosomes. Plasma-seq was consistently able to identify the extra chromosome segments in the trisomy cases, demonstrating the robustness of this technique.

The authors then progressed to whole-genome sequencing of plasma samples from individuals without cancer and those with cancer. These experiments revealed many copy number differences that were previously associated with prostate cancer. Among these changes were the TMPRSS2-ERG fusion, losses of chromosome 8p, gains of chromosome 8q, and gains of the androgen receptor. All of this information was obtained at a very reasonable cost and in only two days. To further demonstrate the effectiveness of plasma-seq, serial plasma analyses were performed on two patients. Of these, one patient had their primary tumor completely removed 13 years prior

Figure 1. An overview of plasma-seq. Blood is drawn from the patient, plasma prepared and the plasma DNA is isolated. The shotgun library is then prepared for sequencing. This step takes a total of 24 hours to perform. Shallow sequencing of the plasma DNA using an Illumina MiSeq machine takes about 12 hours to perform. Finally, alignment of the DNA sequences and analysis of the resulting data take between 2 and 3 hours. bp, base pair; ctDNA, circulating tumor DNA. 
to the plasma analyses. Because the primary tumor was predicted to be heterogeneous, it was divided into six sections and whole-genome sequencing was performed for each region. Consistent with the prediction, wholegenome sequencing revealed different copy number changes in different regions. Plasma samples were taken three different times over a 9-month period, 13 years after tumor resection to analyze secondary tumors in a castration resistant patient. Plasma-seq identified multiple chromosomal rearrangements that had not been present in the original primary tumor. Additionally, the results at each of the three distinct time points were virtually the same, suggesting that one dominant clone released DNA into circulation. These results demonstrate the utility of plasma-seq not only for prostate cancer, but also in tracking changes in any metastatic cancer.

\section{Conclusions}

This is the first whole-genome sequencing analysis from plasma DNA of patients with prostate cancer. Heitzer et al. showed that analysis of plasma DNA from a patient could be used as a noninvasive method of tumor cell genotyping that can be repeated over time and used to monitor tumor responsiveness to therapy. The authors show that plasma-seq is both reliable and robust through various controls and comparisons with other methods. Plasma-seq is faster and cheaper than previously used protocols, while still being reliable, making it more advantageous for use in a clinical setting. It also requires less specialized equipment than what is normally required for circulating tumor cell analysis. One disadvantage of plasma-seq is that the limited coverage makes structural chromosomal rearrangements difficult to identify with high confidence. It also has a reduced resolution for the identification of mutants. This suggests that it is excellent for finding previously identified mutations, but perhaps not as useful for the identification of new mutations involved in prostate cancer metastasis. Given its dramatically reduced cost and time of analysis, plasma-seq will undoubtedly be more widely applied in different clinical settings in the near future.

\section{Abbreviations}

bp, base pair; CRPC, castration-resistant prostate cancer; CTCs, circulating tumor cells; ctDNA, circulating tumor DNA.

\section{Competing interests}

The authors declare that they have no competing interests.

Published: 29 April 2013

\section{References}

1. Damber JE, Aus G: Prostate cancer. Lancet 2008, 371:1710-1721.

2. Jemal A, Siegel R, Xu J, Ward E: Cancer statistics, 2010. CA Cancer J Clin 2010, 60:277-300.

3. Shariat SF, Kattan MW, Vickers AJ, Karakiewicz PI, Scardino PT: Critical review of prostate cancer predictive tools. Future Oncol 2009, 5:1555-1584.

4. Moyer VA; U.S. Preventative Services Task Force: Screening for prostate cancer: U.S. Preventive Services Task Force recommendation statement. Ann Intern Med 2012, 157:120-134.

5. Taylor BS, Schultz N, Hieronymus H, Gopalan A, Xiao Y, Carver BS, Arora VK, Kaushik P, Cerami E, Reva B, Antipin Y, Mitsiades N, Landers T, Dolgalev I, Major JE, Wilson M, Socci ND, Lash AE, Heguy A, Eastham JA, Scher HI, Reuter VE, Scardino PT, Sander C, Sawyers CL, Gerald WL: Integrative genomic profiling of human prostate cancer. Cancer Cell 2010, 18:11-22.

6. Berger MF, Lawrence MS, Demichelis F, Drier Y, Cibulskis K, Sivachenko AY, Sboner A, Esgueva R, Pflueger D, Sougnez C, Onofrio R, Carter SL, Park K, Habegger L, Ambrogio L, Fennell T, Parkin M, Saksena G, Voet D, Ramos AH, Pugh TJ, Wilkinson J, Fisher S, Winckler W, Mahan S, Ardlie K, Baldwin J, Simons JW, Kitabayashi N, MacDonald TY, et al.: The genomic complexity of primary human prostate cancer. Nature 2011, 470:214-220.

7. Olmos D, Brewer D, Clark J, Danila DC, Parker C, Attard G, Fleisher M, Reid AH, Castro E, Sandhu SK, Barwell L, Oommen NB, Carreira S, Drake CG, Jones R, Cooper CS, Scher HI, de Bono JS: Prognostic value of blood mRNA expression signatures in castration-resistant prostate cancer: aprospective, two-stage study. Lancet Onco/ 2012, 13:1114-1124

8. Danila DC, Fleisher M, Scher HI: Circulating tumor cells as biomarkers in prostate cancer. Clin Cancer Res 2011, 17:3903-3912.

9. Forshew T, Murtaza M, Parkinson C, Gale D, Tsui DW, Kaper F, Dawson SJ, Piskorz AM, Jimenez-Linan M, Bentley D, Hadfield J, May AP, Caldas C, Brenton $J D$, Rosenfeld N: Noninvasive identification and monitoring of cancer mutations by targeted deep sequencing of plasma DNA. Sci Trans/Med 2012, 4:136ra168.

10. Heitzer E, Ulz P, Belic J, Gutschi S, Quehenberger F, Fischereder K, Benezeder T, Auer M, Pischler C, Mannweiler S, Pichler M, Eisner F, Haeusler M, Riethdorf S, Pantel K, Samonigg H, Hoefler G, Augustin H, Geigl JB, Speicher MR: Tumor associated copy number changes in the circulation of patients with prostate cancer identified through whole-genome sequencing. Genome Med 2013, 5:30.

doi:10.1186/gm439

Cite this article as: Farris C, Trimarchi JM: Plasma-seq: a novel strategy for metastatic prostate cancer analysis. Genome Medicine 2013, 5:35. 